\title{
Asymmetric Michael Addition Reaction via Rhodium Catalyst Fragmentation
}

\section{Gategory}

Metal-Catalyzed

Asymmetric

Synthesis and

Stereoselective

Reactions

\section{Key words}

dual catalysis

asymmetric Michae addition

chiral Lewis acids

rhodium catalysis

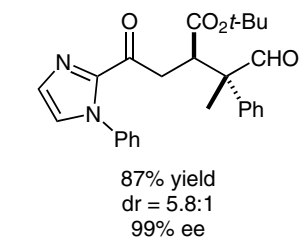


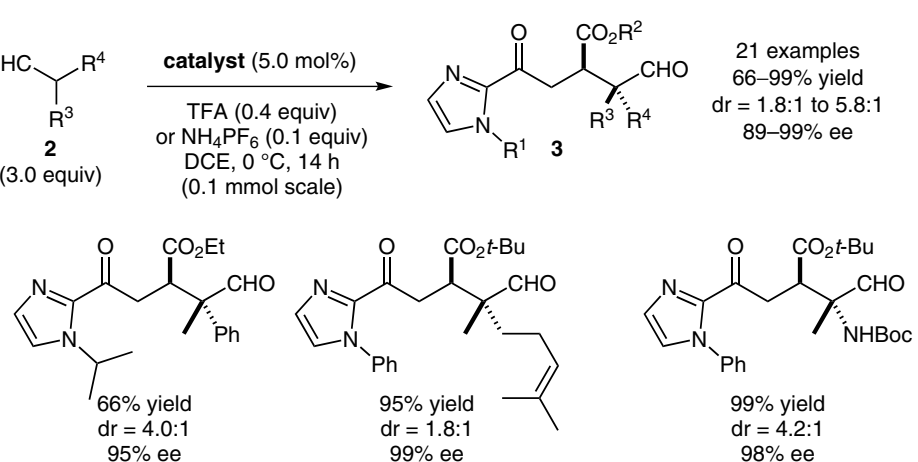

Catalyst synthesis: $\mathrm{RhCl}_{3} \cdot 3 \mathrm{H}_{2} \mathrm{O}$
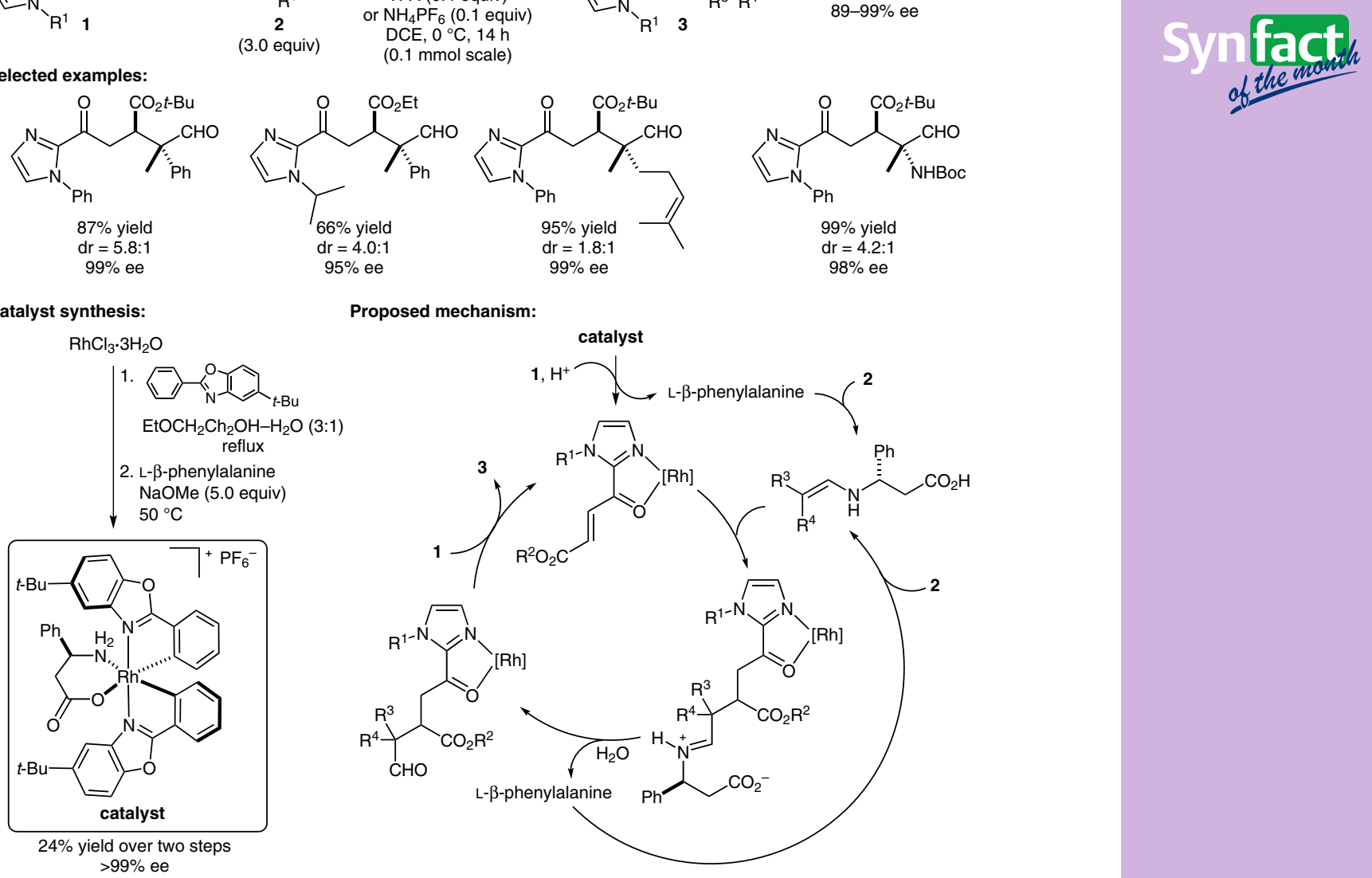

Significance: Dual catalysis has emerged as a powerful approach for the synthesis of enantioenriched compounds. The use of a single catalyst containing two or more functionalities, or the cooperation of two different catalysts, represents the common approach. The authors present an unusual dual catalytic system based on the disintegration of a single chiral rhodium complex in situ. This process was applied in the asymmetric Michael addition of aldehydes to give $\alpha, \beta$-unsaturated 2-acyl imidazoles.
Comment: Reaction between $\alpha, \alpha$-disubstituted aldehydes and $\alpha, \beta$-unsaturated 2-acyl imidazoles was catalyzed by a single chiral rhodium catalyst. The corresponding products were obtained in excellent yields, moderate diastereo- and excellent enantioselectivities. The authors propose a mechanism in which the rhodium catalyst decomposes into a chiral Lewis acid, which activates the 2-acyl imidazole substrate $\mathbf{1}$, and L- $\beta$-phenylalanine, which activates the aldehyde $\mathbf{2}$. 